\title{
Evolution of adiabatic shear bands in zirconium alloy under dynamic deformation
}

\author{
ZOU Dong $\mathrm{Li}^{\mathrm{a}^{*}}$, HE Li Feng ${ }^{\mathrm{b}}$ and XIAO Da Wu \\ China Academy of Engineering Physics, Mianyang 621900, China \\ a*dlzou1980@163.com, bhelifeng@caep.cn, xiaodawu@caep.cn
}

\begin{abstract}
Keywords: Adiabatic shear bands; Zirconium alloy; Dynamic deformation.
\end{abstract}
Abstract. Evolution of adiabatic shear bands in zirconium alloy under dynamic deformation was investigated by means of split Hopkinson pressure bar impacted at different strains and strain rates. The results show that adiabatic shear bands were the main plastic deformation mechanism of zirconium alloy subjected to large strain and high strain rate. With the strain and strain rate increasing, two types of adiabatic shear bands were distinguished: deformed bands and transformed bands. The deformed bands showed the severe strain localization, and the transformed bands mainly composed of the ultrafine and equiaxed grains were confirmed. F, the evolution of adiabatic shear bands in the zirconium alloy under dynamic deformation was proposed.

\section{Introduction}

Zirconium alloy as the fuel cladding materials has been widely used in the nuclear power reactors due to its high melting point, low neutron absorption cross section, good corrosion resistance and excellent mechanical properties. Broad applications of zirconium alloys lead to an increasing tendency that they would be subjected to a dynamic loading or high strain rate loadings inevitably. Thus, it is necessary to achieve fundamental understanding on the deformation and failure mechanisms of zirconium alloy under dynamic loadings. As reported, various mechanical properties [1], corrosion resistance performances [2] and hydrogen absorption properties [3] of zirconium alloy have been investigated extensively. However, the plastic deformation behaviors of zirconium alloy subjected to the dynamic loadings is limited reported. Thus, it is necessary to investigate the dynamic deformation behaviors of zirconium alloy due to fact that the dynamic deformation behaviors of zirconium alloy are closely related with the security of the nuclear power reactors.

Among various post-deformation microstructures, adiabatic shear bands (ASBs) have received much attention due to commonly accepted fact that the ASBs are considered as the precursor of materials damage and failure. The ASBs caused by strain localization have been observed in many materials, and two types of ASBs have been classified: deformed bands (DBs) and transformed bands (TBs). The DBs with black-etching characteristic show the severe strain localization, and the transformed bands with white-etching characteristic mainly compose the ultrafine and equiaxed recrystallized grains. In this paper, the ASBs including the DBs and TBs in zirconium alloy under dynamic loadings are observed and characterized, and the relationship between the ASBs and experimental parameters is discussed.

\section{Experimental}

The annealed $\mathrm{Zr}-4$ alloy plate with thickness of $4.6 \mathrm{~mm}$ was selected as the original material, and the zirconium alloy plate had an equiaxed grains with diameter ranging from 10 to $20 \mu \mathrm{m}$. The cylindrical specimens were cut by electrical discharge machining with $\mathrm{c}$ axis paralleled to normal direction of plate, and the diameter and height of the cylindrical specimens were $6 \mathrm{~mm}$ and $4.6 \mathrm{~mm}$, respectively. Dynamic deformation experiments were conducted on a split Hopkinson pressure bar (SHPB), and the mean strain rate and stress-strain curves were calculated by the one-dimensional stress wave theory. The macroscopic views of the impacted specimens were collected by a scanner, and the microstructure was 
observed by an optical microscope (OM, ZEISS Axiovert 40), a SEM (FEI Nova 400) and EBSD (FEI Nova 400 with a Oxford HKL Channel-5 system) operated at $20 \mathrm{kV}$.

\section{Results and discussion}

Fig.1 shows the stress-strain curve and macroscopic views of zirconium alloy impacted by SHPB at different strains and strain rates. The strain about 0.15 is obtained in zirconium alloy specimen impacted at a strain rate of about $1000 \mathrm{~s}^{-1}$, while the strain about 0.30 is acquired at a strain rate of about $3000 \mathrm{~s}^{-1}$. The strains about 0.50 and 0.70 are obtained in zirconium alloy specimens impacted at a strain rate of about $4500 \mathrm{~s}^{-1}$ and $6000 \mathrm{~s}^{-1}$, respectively. The apparent strain hardening behavior of zirconium alloy can be observed at the strain rate lower than $3000 \mathrm{~s}^{-1}$, and the dramatic drop of the stress can be observed at the strain rate higher than $3000 \mathrm{~s}^{-1}$. The macroscopic views of the specimens deformed at different strains and strain rates are shown in the inset of Fig. 1a. No macro-crack can be observed in the specimens deformed at a strain rate lower than $3000 \mathrm{~s}^{-1}$, and obvious macro-cracks propagated along $45^{\circ}$ shear direction can be observed in the specimens deformed at a strain rate higher than $3000 \mathrm{~s}^{-1}$. Thus, the formation of the macro-cracks in the specimens deformed at a strain rate higher than $3000 \mathrm{~s}^{-1}$ leads to the stress level decreasing dramatically. The cross-section views of the ASBs in zirconium alloy deformed at different strains and strain rates are shown in Fig. 1b, 1c, 1d and 1e. When the specimens are deformed at a strain 0.15 and strain rate of about $1000 \mathrm{~s}^{-1}$, no bands are observed. When the strain and strain rate increases to 0.30 and $3000 \mathrm{~s}^{-1}$, only DBs can be observed. The DBs are mainly located at four corners of the specimen, propagating along $45^{\circ}$ shear direction from the corners to the deep matrix. No ASB or strain localization is detected in other areas. When the specimens are deformed at a strain 0.50 and strain rate of about $4500 \mathrm{~s}^{-1}$, both DBs and TBs are observed on the specimen cross-section. The DBs are located at the specimen corner, and the TBs are located at the tip of the cracks. When the strain and strain rate increases to 0.70 and $6000 \mathrm{~s}^{-1}$, the quantities of both DBs and TBs on the specimen cross-section increase obviously, indicating that the ASBs play an important role for the plastic deformation of zirconium alloy subjected to large strain and high strain rate loadings.

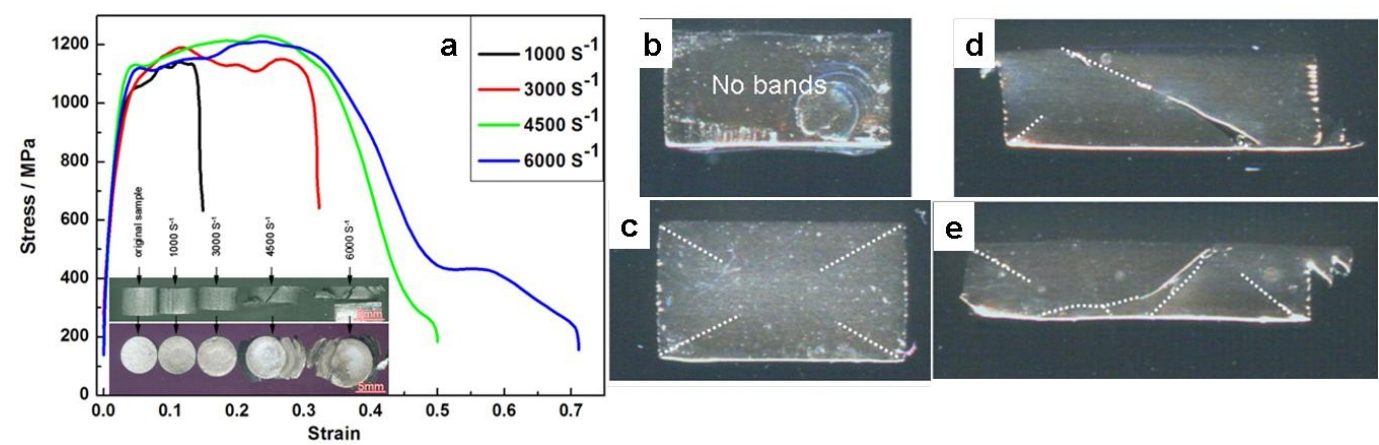

Fig. 1 Stress-strain curve and macro-views of zirconium alloy impacted by SHPB at different strains and strain rates: (a) stress-strain curve; (b) strain: 0.15 , strain rate: $1000 \mathrm{~s}^{-1}$; (c) strain: 0.30 , strain rate: $3000 \mathrm{~s}^{-1}$; (d) strain: 0.50 , strain rate: $4500 \mathrm{~s}^{-1}$; (e) strain: 0.70 , strain rate: $6000 \mathrm{~s}^{-1}$

SEM images of the ASBs in zirconium alloy deformed at different strains and strain rates are shown Fig. 2. The DBs formed at a strain of 0.30 and strain rate of $3000 \mathrm{~s}^{-1}$ show the severe strain localization, and the severe deformed and elongated grains in the deformed bands can be observed. The TBs formed at a strain of 0.50 and strain rate of $4500 \mathrm{~s}^{-1}$ compose of the ultrafine and equiaxed grains. Compared with the DBs, the boundary between the TBs and the matrix is obvious, and almost no transition zone from the TBs to the matrix can be observed. Fig. 3 shows EBSD images of DBs in zirconium alloy deformed at a strain of 0.30 and strain rate of $3000 \mathrm{~s}^{-1}$. The EBSD indexed zone of DBS is shown in Fig. $3 \mathrm{a}$, and the corresponding post-processed images of the indexed zone are shown in Fig. 3b and 3c. It can be seen that the DBs are located at the center of the EBSD indexed zone. Compared with the neighboring matrix, the indexed rate in the DBs is much lower. Severe plastic deformation and high internal stress in the DBs lead to the indexed rate decreasing. 
EBSD index of the TBs in zirconium alloy deformed at a strain of 0.70 and strain rate of $6000 \mathrm{~s}^{-1}$ is shown in Fig. 6. It can be seen that the TBs are located at the center of the indexed zone. Compared with the grains in the matrix with diameter ranging from 10 to $20 \mu \mathrm{m}$, the grain size in the TBs decreases two orders of magnitude. The transition from the coarse grains in the matrix to the fine grains in the TBs is distinct, and the boundary between the matrix and the TBs is clear. The diameter of the grains in the transformed bands is not uniform, and the gradient variation $\mathrm{f}$ the grains from the boundary to the center of the TBs can be observed, which should be associated with temperature distribution in the TBs. The gradient temperature distribution across the ASBs has been calculated [4], and the maximum temperature rising at the center of the ASBs was revealed, leading to the grains at the center of the TBs finer than other zones. The ultrafine grains with high angle boundaries demonstrate that the formation of the ultrafine grains in the TBs should be a result of the dynamic recrystallization. The formation mechanism of the ultrafine and equiaxed grains in the TBs in zirconium alloy has been proposed by Meyers [5]. The calculated thermodynamics and dynamics suggested that the rotational dynamic recrystallization mechanism was considered as a reasonable model to explain the formation of the ultrafine grains in the TBs. The detailed rotational dynamic recrystallization model can be seen in reference [6].

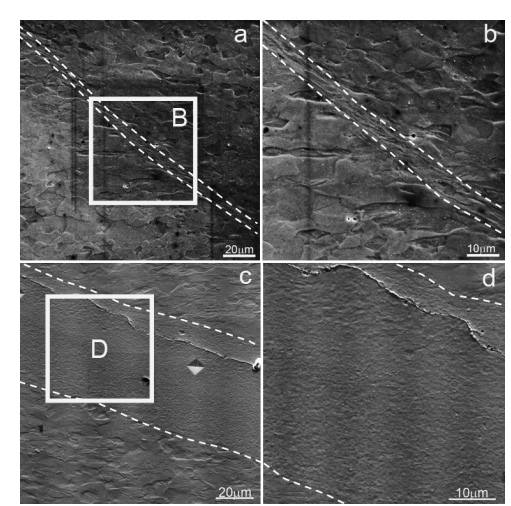

Fig. 2 SEM images of the ASBs in zirconium alloy impacted by SHPB at different strains and strain rates: (a) strain: 0.30 , strain rate: $3000 \mathrm{~s}^{-1}$; (b) high magnified image in zone $\mathrm{B}$; (c) strain: 0.50 , strain rate: $4500 \mathrm{~s}^{-1}$; (d) high magnified image in zone D

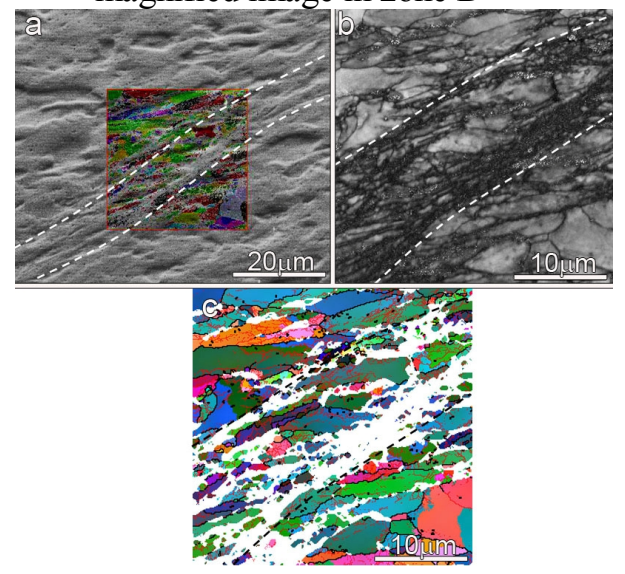

Fig. 3 EBSD analysis of the DBs in zirconium alloy impacted by SHPB at a strain of 0.30 and strain rates of 3000 $\mathrm{s}^{-1}:$ (a) indexed zone; (b) band contrast map; (c) all Euler map

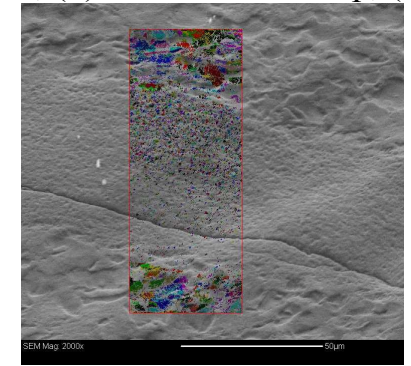

Fig. 4 EBSD index of the TBs in zirconium alloy impacted by SHPB at a strain of 0.50 and strain rates of $4500 \mathrm{~s}^{-1}$ 
As reported [7], the critical strain and strain rate for the formation of the DBs and TBs were different, and high critical strain and strain rate were required for the formation of the TBs. Thus, the TBs are regarded as the result of the further development of the DBs. The formation process of the DBs and TBs in zirconium alloy can be depicted as follows. At low strain and strain rate, the prismatic and pyramidal slips are proposed as the primary plastic deformation mechanism of the zirconium alloy. As the strain and strain rate increase to 0.30 and $3000 \mathrm{~s}^{-1}$, the stress concentration at the corner of the specimen leads to the strain localization to form the DBs. At this time, the severe deformed and elongated grains are formed in the DBs due to fact that the temperature rising caused by plastic deformation in the DBs is not high enough to induce the occurrence of the dynamic recrystallization. When the strain and strain rate increase to 0.50 and $4500 \mathrm{~s}^{-1}$, the severe plastic deformation is concentrated into materials in the DBs, leading to the localized shear strain in the DBs increasing. When the critical localized strain and strain rate in the DBs is reached, the severe deformed grains assisted with high plastic temperature rising leads to the occurrence of the dynamic recrystallization, and the severe deformed and elongated grains are developed into the ultrafine and equiaxed grains, while the DBs are evolved into the TBs.

\section{Summary}

1. Adiabatic shear bands are the main plastic deformation mechanism of zirconium alloy subjected to large strain and high strain rate.

2. Two types of adiabatic shear bands are distinguished The deformed bands show the severe localized plastic deformation, and the transformed bands mainly composed of the ultrafine and equiaxed grains.

3. The evolution of adiabatic shear bands in the zirconium alloy is proposed. With the strain and strain rate increasing, the deformed bands would be evolved into transformed bands.

\section{Acknowledgements}

Thanks for the support of the SUOZHANG Foundation (SJ201309) and National Natural Science Foundation (51401187).

\section{References}

[1] F. Xu, R.A. Holt, M.R. Daymond, Modeing lattice strain evolution during uniaxial deformation of textured zircaloy-2, Acta Mater. 56 (2008) 3672-3687.

[2] J.H. Baek, Y.H. Jeong, I.S. Kim, Effects of the accumulated annealing parameter on the corrosion characteristics of a Zr-0.5Nb-1.0Sn-0.5Fe-0.25Cr alloy, J. Nucl. Mater. 280 (2000) 235-245.

[3] H.G. Kim, J.Y. Park, B.K. Choi, Y.H. Jeong, Evaluation of pre-transition oxide on Zr-0.4Nb alloy by using the HVEM, J. Nucl. Mater. 374 (2008) 204-210.

[4] X.B. Wang, Temperature distribution in adiabatic shear band for ductile metal based on Johnson-Cook and gradient plasticity models, Trans. Nonferrous Met. Soc. China, 16 (2006) 333-338.

[5] B.K. Kad, J.-M. Gebert, M.T. Perez-Prado, M.E. Kassner, M.A. Meyers, Ultrafine-grain-sized zirconium by dynamic deformation, Acta Mater. 54 (2006) 4111-4127.

[6] M.A. Meyers, V.F. Nesterenko, J.C. Lasalvia, Q. Xue, Shear localization in dynamic deformation of materials: microstructural evolution and self-organization, Mater. Sci. Eng. A, 317 (2001) 204-225.

[7] Y.B. Xu, J.H. Zhang, Y.L. Bai, M.A. Meyers, Shear localization in dynamic deformation: microstructural evolution, Metall. Mater. Trans. A 39 (2008) 811-843. 